\title{
Visible Light, Wide-Angle Graded Metasurface for Back Reflection
}

\author{
Nasim Mohammadi Estakhri, ${ }^{\dagger}$ Verena Neder, ${ }^{\ddagger} \S$ Mark W. Knight, ${ }^{\S}$ Albert Polman, ${ }^{\ddagger}$ and Andrea Alù ${ }^{*},{ }^{\dagger}$ \\ ${ }^{\dagger}$ Department of Electrical and Computer Engineering, The University of Texas at Austin, Austin, Texas 78712, United States \\ ${ }^{\ddagger}$ Institute of Physics, University of Amsterdam, Science Park 904, 1098 XH Amsterdam, The Netherlands \\ ${ }^{\S}$ Center for Nanophotonics, AMOLF, Science Park 104, 1098 XG, Amsterdam, The Netherlands
}

\section{Supporting Information}

ABSTRACT: Metasurfaces, or phase-engineered quasi-2D interfaces, enable a large degree of control over the reflection, refraction, and transmission of light. Here we demonstrate the design and realization of a visible light gradient metasurface tailored for highly efficient back reflection based on the Huygens-Fresnel principle. The metasurface emulates the functionality of a Littrow grating, capable of efficiently channeling light into the first negative Floquet order over a broad angular range and bandwidth at visible frequencies. Our theoretical results predict unitary efficiency for extremely low profiles and an optical response that is robust against discretization and design modifications. The experimentally realized metasurface is comprised of high-index $\mathrm{TiO}_{x}$ nanowires over a protected $\mathrm{Ag}$ mirror, enabling back reflection with efficiency above $85 \%$ in the visible range, close to the reflectivity of the bare silver mirror. The presented analytical design methodology and the resulting low-profile device are advantageous compared to conventional gratings, while offering broadband efficiencies over a range of incidence angles.

KEYWORDS: gradient metasurfaces, back reflector, dielectric metasurfaces, gratings, nanophotonics
$G$ radient metasurfaces have started to significantly influence modern optical technology, as they offer the possibility of replacing a wide range of bulky optical components with low-profile and low-loss patterned surfaces. $^{1-4}$ Graded metasurfaces rely on nanostructured surfaces with controlled variation of their transverse impedance, enabling a plethora of operational possibilities, including anomalous wave refraction and reflection, ${ }^{5,6}$ holography, ${ }^{7,8}$ cloaking, $^{9-11}$ focusing and beam shaping, ${ }^{5,12}$ polarization management, ${ }^{13,14}$ and nonreciprocal beam control when combined with time modulation. ${ }^{15,16}$ The nanoscale control of strong wave-matter interactions in metasurfaces has opened remarkable opportunities for low-loss, integrable planar optics. ${ }^{2}$ In contrast to other ultrathin configurations based on resonantly excited elements, ${ }^{17-21}$ gradient metasurfaces typically rely on the response of a combination of nonresonant scatterers and their tailored arrangement, enabling this class of metasurface to support inherently broadband and robust optical responses that are resilient to losses. ${ }^{14}$ In this work, we apply the concept of graded metasurfaces to design broadband back reflectors, based on distributed surface impedances. Back reflectors are a class of periodic structures that are specifically tailored to funnel the incident energy into the first negativeorder Floquet harmonic. These structures are traditionally implemented using binary patterns over finite thicknesses, ${ }^{19,22-24}$ which typically need to be optimized to maximize the coupling efficiency to the backward Floquet order $s_{1}^{-24,25}$ Here we use an alternative method based on the
Huygens-Fresnel principle to define the ideal surface impedance profile of ultrathin metasurfaces that enables arbitrary negative reflection. The ideal operation of the designed surface is achieved by controlling the required secondary scattering sources on the metasurface to reconstruct the desired Floquet order. This approach offers flexibility in design, avoiding the need for optimization or specific material parameters, and enables high back-reflection efficiencies over the desired range of angles of incidence (AOI), angles of reflection, and frequency. In order to validate our theoretical approach, we employ high-resolution nanolithography to experimentally implement a back-reflecting graded metasurface that operates in the visible range.

\section{RESULTS AND DISCUSSION}

First, we focus our study on the design of an ideal gradient metasurface that reflects all the impinging energy back to the source for a specific frequency and angle for linear polarized light, essentially operating as a zero-thickness Littrow grating. This functionality is equivalent to an ideal retroreflector for this frequency and AOI (Figure 1A shows the concept and a schematic of the fabricated structure). While an ideal homogeneous mirror (Figure 1B) reflects the impinging light toward the specular direction due to momentum conservation, this ideal metasurface with a tailored gradient of the reflection

Received: December 4, 2016

Published: January 23, 2017 
A

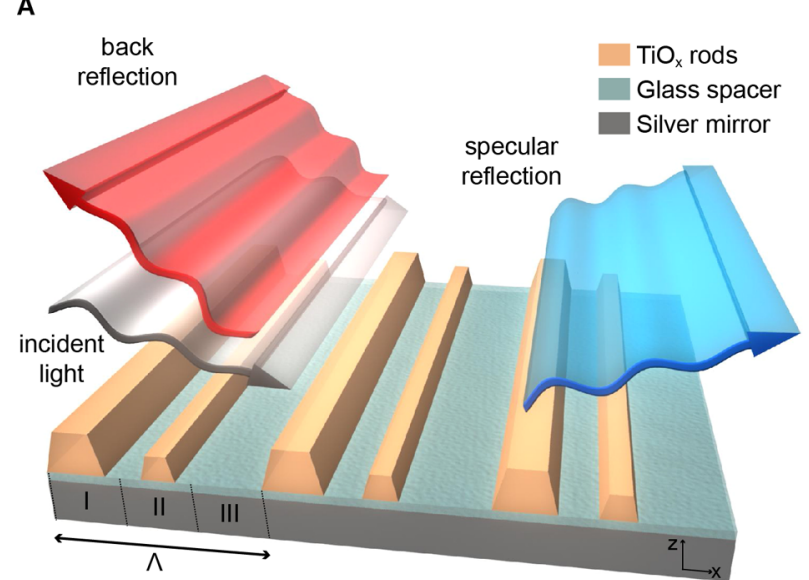

B

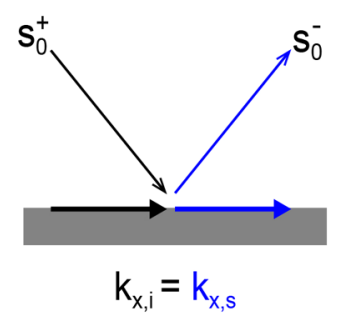

C

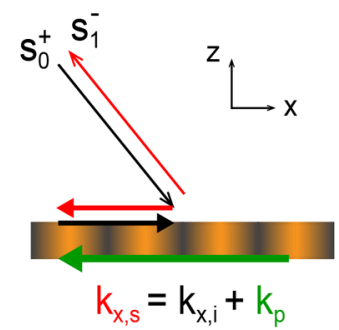

Figure 1. Operation principle of a metasurface back reflector. (A) Schematic illustration of back reflection from a gradient metasurface. (B) Representation of specular reflection from an ideal mirror: incident light $\left(s_{0}^{+}\right.$, black $)$is specularly reflected $\left(s_{0}^{-}\right.$, blue $)$from an ideal mirror due to momentum conservation at the interface. (C) Scattering channels for reflection from a gradient metasurface: incident light is reflected back $\left(s_{1}^{-}\right.$, red) toward the source due to the transverse momentum imparted by the inhomogeneous interface. The additional negative momentum $k_{\mathrm{p}}$ (green) is introduced by a tailored gradient of the reflection phase. Momenta in the $x$-direction are shown by $k_{x, \mathrm{i}}$ and $k_{x, \mathrm{~s}}$ for incident and reflected waves, respectively.

phase (Figure 1C) can impart a suitable additional negative transverse momentum to the impinging wave and reflect the entire impinging light flux back to the source.

Following the approach introduced in refs 5 and 12 and tailored to efficiently manipulate reflected beams in ref 14 , we derive in the Methods section the analytical expression of the local reflection coefficient that an ultrathin metasurface needs to support in order to achieve single-angle retroreflection with unity efficiency for illumination angle $\theta_{0}$ in the $x-z$ plane, where $\hat{z}$ is the direction normal to the surface:

$$
r(x)=\frac{-1+\cos \theta_{0}-\mathrm{e}^{2 \mathrm{i} \pi x / \Lambda}\left(1+\cos \theta_{0}\right)}{-1-\cos \theta_{0}+\mathrm{e}^{2 \mathrm{i} \pi x / \Lambda}\left(-1+\cos \theta_{0}\right)}
$$

The required reflection coefficient is unitary all across the surface, implying that it can be achieved with a fully passive interface with inhomogeneous phase profile $\varphi(x)$, shown in Figure $2 \mathrm{~A}$ for $\theta_{0}=35.7$ degrees, with period $\Lambda=\frac{\lambda}{2 \sin \theta_{0}}$ where $\lambda$ is the wavelength of operation in free space. In general, the optimal reflection coefficient of a gradient metasurface may also involve local amplitude modulation. ${ }^{26}$ At the retroreflection angle, however, the exact impedance matching between the incident and scattered beam guarantees that $100 \%$ wave conversion is accessible using a purely lossless surface profile, as described by the local phase variations in eq 1 . This
A
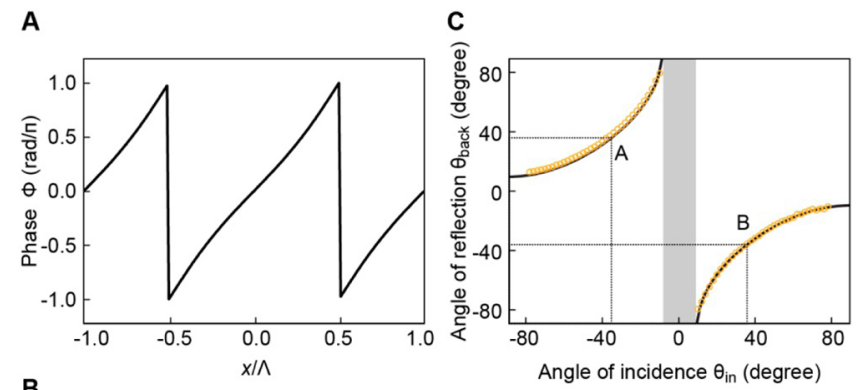

B

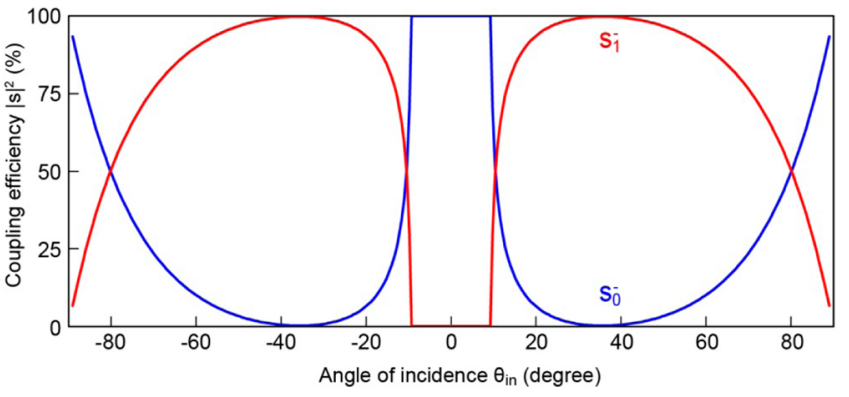

Figure 2. Wide-angle operation of an ideal back-reflector metasurface. (A) Calculated local phase profile of the ideal surface $\phi(x)=\angle r(x)$, designed for retroreflection at AOI $\theta_{0}=35.7$ degrees following eq 1 , with a surface period $\Lambda=\lambda /\left(2 \sin \theta_{0}\right)$. (B) Numerically calculated coupling efficiency of the ideal surface in panel A for different incident angles and for s-polarized illumination. Blue and red curves show the percentage of power coupled toward the specular direction $\left(s_{0}^{-}\right)$and first diffraction order $\left(s_{1}^{-}\right)$, respectively. (C) Calculated (solid black line) and measured (yellow circles) angular dispersion of the gradient surface for the \pm 1 diffraction orders. The black lines correspond to the ideal surface in panel $\mathrm{A}$, and the yellow circles are analogous results measured at $\lambda=700 \mathrm{~nm}$ for the fabricated sample. A and B correspond to the ideal retroreflection points where $\theta_{\text {back }}=-\theta_{\text {in }}= \pm 35.7$ degrees (Littrow configuration). Inside the highlighted gray region, the nonspecular diffraction orders are evanescent.

nonlinear phase profile exactly compensates for the momentum mismatch between the incoming and the desired retroreflected waves (Figure 1C). For values of incident wave different from $\theta_{0}$, the reflected wave does not align with the incident wave and the structure operates as an ultrathin back reflector, redirecting light back into the half-space of incidence. Compared to previous attempts to realize highly efficient back-reflecting gratings, this approach is fully analytical, where calculations based on the Huygens-Fresnel principle allow us to engineer the scattering pattern over an infinitesimally thin surface at will and without requiring iterative optimization. ${ }^{26,27}$ For instance, the surface may be equivalently designed to couple a desired portion of the impinging power toward the specular reflection, and the other portion toward back reflection. This concept is also fully extendable to two-dimensional surfaces and arbitrary polarization control.

For an ideal continuously modulated metasurface with a local reflection given by eq 1 , given the periodicity of the modulation, the reflected power can couple to only two propagating diffraction orders, the specular reflection $s_{0}^{-}$and the first negative Floquet order $\overline{s_{1}^{-}}$. The numerically calculated coupling efficiency to these orders as a function of illumination angle is shown in Figure 2B for the surface with phase profile in Figure 2A (see Supplementary Note 1). As expected, we obtain $100 \%$ coupling efficiency at $\theta_{0}=35.7$ degrees (the design angle), i.e., $\left|s_{1}^{-}\right|^{2}=1$. For this AOI, the metasurface operates in the Littrow configuration and the angle of reflection equals 35.7 
A

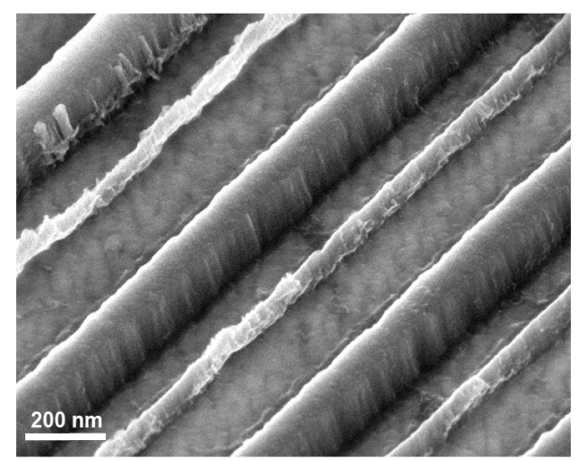

C

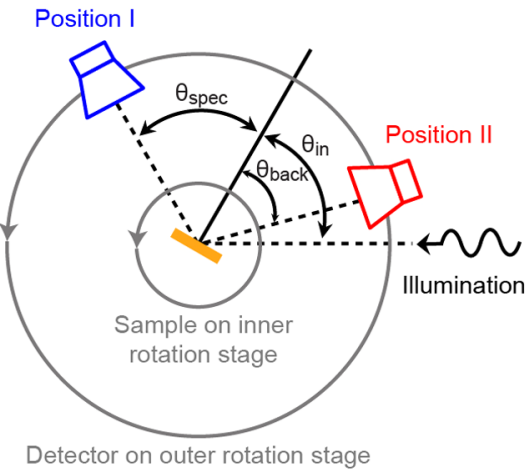

B

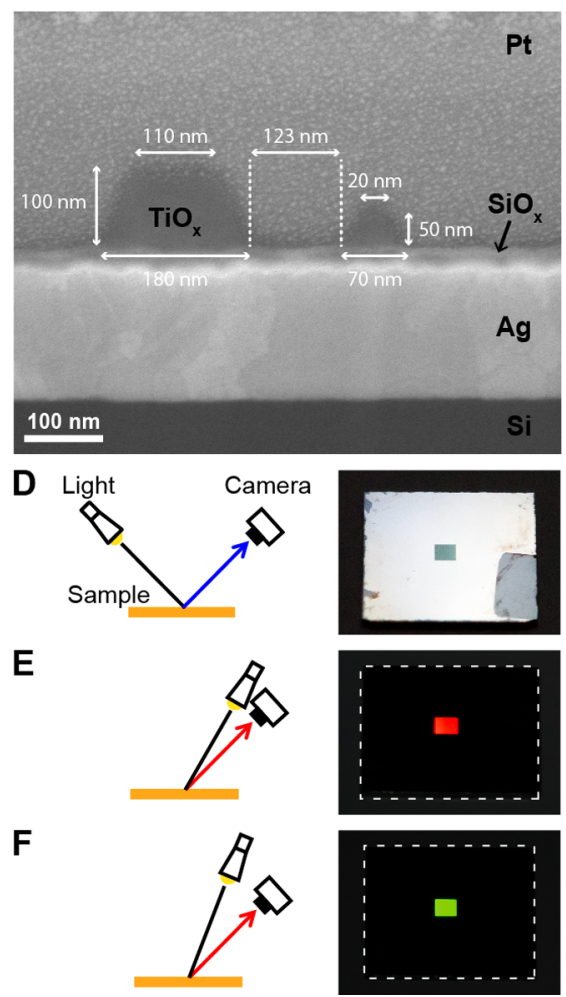

Figure 3. Fabricated structure and measurement setup. (A) SEM image (top view, under $40 \%$ tilt) and (B) cross section of the fabricated sample with extracted dimensions of the device. One unit cell of the structure is composed of three regions: two $\mathrm{TiO}_{x}$ nanorods and the bare mirror. See Methods section for detailed geometry information. The Pt layer on top of the sample was added in the cross-section fabrication process to get a clean cross-section. (C) Schematic of the measurement setup: $\theta_{\text {in }}$ can be changed by rotating the sample on the inner rotation stage while the illumination arm is kept fixed. The coupling intensity to the different diffraction orders is measured by independently rotating the detector on the outer rotation stage to position I to measure $\theta_{\text {spec }}$ or position II to measure $\theta_{\text {back }}$. Illumination and detection planes are slightly tilted horizontally to allow retroreflection measurements without blocking the illumination. (D-F) Photographs of the fabricated structure on the right $\left(1.5 \times 1.5 \mathrm{~mm}^{2}\right.$ square in the center of the $12 \times 12 \mathrm{~mm}^{2}$ silver mirror, bare Si residual from fabrication process in the lower-left corner) and schematic of photography setup on the left. Panel D shows the specular response under illumination from the back with a commercial flashlight: no light is reflected in the specular direction from the structure (dark square in the middle). Panels E-F show efficient back reflection of the sample when illuminated with a white light lamp for different angles. The angle between light and camera was increased in F compared to E.

degrees. In addition, the figure shows that the angular response is robust. For an AOI range $11<\theta_{\text {in }}<80$ degrees over half of the incident power is redirected into the nonspecular direction, despite these angles residing outside of the Littrow design parameters. This broad angular response is associated with the fact that the momentum imparted by the surface does not change with the incidence angle, ${ }^{5}$ and it is sufficiently negative to ensure that the angle of the emerging reflected beam stays negative over a broad angular range. In general, the attainable angular range may be further increased by designing the retroreflection angle close to 45 degrees. The back-reflection angle varies as a function of impinging angle following the grating equation for first-order diffraction $\theta_{\text {back }}=\sin ^{-1}\left(\sin \theta_{\text {in }} \mp \frac{\lambda}{\Lambda}\right)$, where the $\mp$ sign refers to $\theta_{\text {in }}>0$ and $\theta_{\text {in }}<0$, respectively, as plotted in Figure 2C. The lower cutoff for $\theta_{\text {in }}=11$ degrees is simply determined by the cutoff of $s_{1}^{-}$for close-to-normal incidence, and it can be adjusted by design.

An interesting feature evident in Figure $2 \mathrm{~B}$ is the perfectly symmetric response of the back-reflective surface. The symmetry arises despite the fact that the geometric profile of the surface, described by eq 1 , is asymmetric and tailored for a specific oblique illumination. This symmetry is a general result stemming directly from reciprocity, ${ }^{28,29}$ and it is not limited to our particular configuration. More specifically, if the surface is designed to back reflect with $x \%$ efficiency for the AOI $\theta_{\mathrm{i}}$, it ensures $(100-x) \%$ coupling to the specular direction $\left(\theta_{\mathrm{r}}=\theta_{\mathrm{i}}\right)$. Reciprocity then ensures that, when the direction of illumination is flipped and the surface is illuminated from the specular direction, exactly $(100-x) \%$ of the power can be coupled back toward $\theta_{i}$. The remaining $x \%$ of the power must be scattered through the only remaining scattering channel, which is the corresponding back reflection. Given that the period is unaffected by the incidence direction and it is designed to admit only two diffraction orders, all remaining energy $(x \%)$ will be coupled to the back-reflected beam. Thus, the blue curve in Figure $2 \mathrm{C}$ is centrosymmetric, and with the broad angular response in the negative half-plane, reciprocity ensures an equally broad response for positive incidence angles.

The properties described so far are desirable from a practical standpoint, indicating that there is no trade-off between directionality and efficiency in the proposed metasurface, and back reflection can be achieved over a broad range of angles, even far from normal incidence. This property also suggests an interesting possibility for applications in the retroreflection industry. While the designed surface is not a true retroreflector, for detectors using narrowband illumination and a relatively 
wide collection aperture this surface may reflect nearly all of the impinging energy back to the source. Another interesting application of this surface might be replacing the diffusive retroreflective tapes (over a limited angle), as the efficiency of reflection is significantly larger than available reflective tapes and the metasurface can be tailored to operate for an extremely broad AOI range. ${ }^{30}$

In order to practically realize the proposed metasurface, we need to discretize the ideal phase profile in eq 1. Assuming an equal discretization of the ideal phase profile into $N$ phase steps, the coupling efficiency to the retroreflected order gets closer to $100 \%$ as the number of steps increases (Supplementary Note 2). Interestingly, even a coarse discretization with only two discretization steps yields a retroreflection efficiency larger than $75 \%$. The reason behind this robustness is again associated with the nature of our design. First, the diffraction phenomenon is nonresonant and therefore inherently robust to perturbations. Second, the period $\Lambda$ determines two propagating diffraction orders for the operational frequency, ensuring that, as long as the overall phase gradient along each unit cell suppresses the coupling toward the zerothorder diffraction (specular reflection), reflection will be funneled toward the first order in the back direction.

The ultrathin profile of the designed surface provides an ideal platform for microwave or terahertz graphene-based metasurfaces, which are inherently deeply subwavelength. Here, we chose to realize the device characterized in Figure 2A using a nanostructured dielectric metasurface operating in the visible spectrum (Figure 3A,B) with subwavelength thickness $t=100$ $\mathrm{nm}$, made of $\mathrm{TiO}_{x}$ trapezoidal bars on top of a $\mathrm{Ag}$ mirror via ebeam lithography and evaporation (see Methods for a description of the fabrication process and Figure 1A for a schematic of the fabricated structure). The dielectric nature of $\mathrm{TiO}_{x}$ and its relative high index $(n=2.35$ for $\lambda=500 \mathrm{~nm}$ to $n=$ 2.23 at $\lambda=1000 \mathrm{~nm}$ ) are suitable to minimize absorption and provide reasonable phase control over a relatively small thickness. $^{31}$ We designed the structure with three phase discretization steps, $N=3$, for operation at $\lambda=700 \mathrm{~nm}$, tailored for s-polarized excitation (electric fields lies in the metasurface plane). The variation of the local reflection coefficient in the first two elements is achieved by controlling the geometry of the nanorods. The third segment consists of the bare back-mirror (Figure 3A).

The fabricated structure was excited with a weakly converging beam such that the excitation angle was welldefined. The reflected intensity was measured using an optical power meter (see Methods section for more details on the measurement). The sample was mounted in the center and the power meter on the outer ring of a rotating stage, while the illumination direction was held constant. This enabled independent control of excitation and sampling angles, as depicted in the schematic of the measurement setup in Figure 3C. We chose $\Lambda=600 \mathrm{~nm}$ (605 nm in the fabricated surface) to enable efficient back reflection in the free-space wavelength range $\lambda=490-940 \mathrm{~nm}$.

The previous analysis ensures that an efficient back reflector can be implemented over a deeply subwavelength thickness. At shorter wavelengths, and specifically over the visible spectrum, current nanofabrication techniques limit the accessible resolution and accuracy of sub-hundred-nanometer features. To reduce the sensitivity of the device to nanofabrication errors, we chose a thickness of $t=100 \mathrm{~nm}$ for the surface. The efficient operation of our fabricated sample from the practical observer standpoint is visualized in Figure 3D-F. Photographs of the sample can be seen next to schematics of the photography setup. In Figure 3D the bright specular reflection of the Ag mirror around the structure is visible, while the dark square in the middle of the sample where the metasurface is placed indicates that specular reflection is almost absent. In contrast, the center of the sample is noticeably bright for an observer sitting close to the excitation source, as can be seen in Figure 3E,F for different incoming angles. The bright color that can be observed in back reflection depends on the angle of observation and illumination. As we mentioned previously, these pictures also indicate the possibility of utilizing these surfaces as efficient ultrathin retroreflectors for AOI close to the retroreflection angle. In this case, while the observable color of the reflected wave varies, the mirror covered with the designed metasurface is noticeably brighter than a conventional mirror.

The optical response of the fabricated sample is quantitatively demonstrated in Figure 4. Symmetric scattering resulting
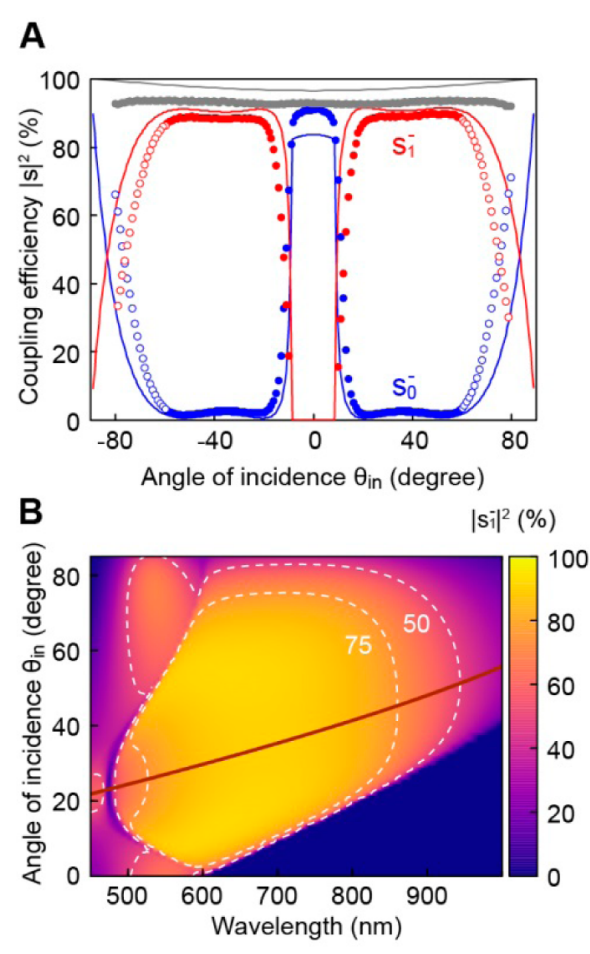

Figure 4. Back reflection response of the fabricated device. (A) Angular response at $\lambda=700 \mathrm{~nm}$. Comparison between measurements (circles) and numerical simulations (solid lines). Coupling efficiencies for the specular reflection $s_{0}^{-}$and the first-order negative reflection $s_{1}^{-}$ are shown with blue and red colors, respectively. The empty circles indicate reflection measurements for angles above $\left|\theta_{\text {in }}\right|=60$ degrees, for which the spot size of the beam exceeds the structured area and part of the beam is specularly reflected by the mirror next to the structure. The measurements and simulations of the bare mirror are depicted in gray. The homogeneous surface supports specular reflection with approximately $10 \%$ absorption across all angles. (B) Numerical simulation results of the angular/frequency dispersion of the structure with the fabricated dimensions, showing the coupling efficiency toward the first-order negative reflection $s_{1}^{-}$and highlighting the $75 \%$ power and $50 \%$ power operation regions. The dark red line indicates the ideal retroreflective loci (Littrow configuration), for which the incoming and the reflected wave are aligned. More than $50 \%$ back reflection is achieved across $\lambda=490-940 \mathrm{~nm}$ and $\theta_{\text {in }}=24-51$ degrees. 
from reciprocity permits a complete characterization of the device while scanning only half the angular range (Figure 4A). However, to confirm the theoretical results, we performed measurements over the full range of angles. In the figure, we compare the specular reflection to the measurements obtained using a flat silver mirror, similar to the ground plane utilized in our device, allowing a direct comparison that provides a quantitative calibration of the measured efficiency. The gray circles in Figure 4A present the measured angular response of the silver mirror when illuminated with s-polarized light at $\lambda=$ $700 \mathrm{~nm}$. We observe that around $10 \%$ of the incident power is lost, either through absorption or diffuse scattering. The measured response of the silver mirror is slightly lower than simulated reflectance using the tabulated dielectric response of single-crystalline silver (Figure 4A). This difference is attributed to nanoscale surface roughness, resulting from evaporation and other fabrication defects.

The level of back reflection of our sample is shown with red circles in Figure 4A, demonstrating that most of the scattered light is indeed efficiently back reflected. The specular reflection thus significantly drops over a wide angular region around the retroreflective angle $\theta_{0}=35.7$ degrees. The scattered power is focused toward the backward diffraction channel $\left(s_{1}^{-}\right.$in Figure $1 C)$, yielding a coupling efficiency of $88 \%$ under illumination at $\theta_{0}=35.7$ degrees and with less than $10 \%$ of the impinging power being absorbed or diffusely scattered at the design frequency under illumination from all angles, except around the Wood's anomaly, consistent with the absorption levels obtained from the bare silver back-mirror. We note that the presence of the plasmonic mirror creates additional absorption at the metal-dielectric interface. For applications requiring the highest efficiencies, such as pulse shaping, stretching, and compression, the Ag mirror could be replaced with an optimized Bragg reflector.

Although the metasurface was originally designed for an AOI of 35.7 degrees, increasing the incidence angle toward grazing angles does not significantly affect the overall efficiency, and up to a remarkably large angle $\left|\theta_{\text {in }}\right|=75$ degrees, at $\lambda=700 \mathrm{~nm}$ the dominant portion of the scattered power remains in the same half-plane of the incident wave. For illumination at angles less than $\left|\theta_{\text {in }}\right|<9$ degrees, the second scattering channel is nonradiative for $\lambda=700 \mathrm{~nm}$, and thus it is not excited (Figure $2 \mathrm{C})$. Over this range, the metasurface operates as a simple mirror, as observed in Figure 4A. For comparison, the solid lines in Figure 4A also show the calculated coupling to the two scattering orders obtained using full-wave simulation for a structure with the same geometry as the fabricated device (see Methods section for details about simulation). The simulated results agree well with our experiment, even though a slightly lower cutoff at large angles is observed in the measured data compared to the calculated curves. This is due to the small size of our sample, as the area where the measurement beam hits the structure increases with higher incoming angles and exceeds the structure area for $\left|\theta_{\mathrm{in}}\right|>60$ degrees. In this angular range, part of the light is specularly reflected from the bare mirror adjacent to the metasurface.

A remarkable property of the back-reflecting metasurface consists in its broadband operation, which is attributed to the inherent stability of retroreflection as well as the nonresonant nature of the involved scattering phenomenon, based on the phase gradient imposed through the metasurface. While the surface impedance of the trapezoids is expected to change over frequency, its relative slope across each unit cell is not significantly affected by frequency variations, ensuring that the net momentum imparted to the impinging wave still funnels most of the energy back to the first negative diffraction order. To further investigate this property, we determined the amplitude dispersion of the coupling to the two scattering modes for a structure with the dimensions of the fabricated device through full-wave simulations for all incident angles (Figure 4B). As expected, the designed metasurface operates over an extremely broad half-power wavelength range $\lambda=490-$ $940 \mathrm{~nm}$ in terms of single-angle retroreflection efficiency. We verified our simulations with experimental measurements at multiple wavelengths, in addition to the $\lambda=700 \mathrm{~nm}$ case, as reported in the Supplementary Note 3. It is worth mentioning that the angular range and bandwidth of the grating may be further increased through optimization; however, here we presented a configuration derived directly by our analytical model in eq 1, also including all fabrication tolerances (e.g., height of second rod is smaller than $t=100 \mathrm{~nm}$ ). The robustness of this design against minor fabrication errors is demonstrated numerically in Supplementary Note 4.

The response of the designed metasurface is also controllable with the polarization of the incident wave. The surface features utilized for this implementation are inherently anisotropic, as their scattering properties depend on the orientation of the field vectors, and the local reflection phase is approximately constant for p-polarized illumination. Consequently, the surface operates as a simple mirror for this polarization, as discussed and verified in Supplementary Note 5. In other words, this metasurface selectively reflects in opposite half-planes the two impinging polarizations. We are currently investigating polarizationindependent designs of back-reflecting metasurfaces based on a similar principle, achieved by considering $2 \mathrm{D}$ arrangements of isotropic surface elements. ${ }^{13,14}$

\section{CONCLUSION}

We have discussed the design principle and advantages in terms of size, simplicity of design and fabrication, bandwidth, acceptance angular range, low profile, and efficiency of gradient metasurfaces for back-reflecting surfaces (i.e., ultrathin Littrow gratings). Indeed, we have shown that there is no fundamental limitation on the efficiency or size of a Littrow grating. One of the challenges in experimentally realizing the optimal surface profile derived in eq 1 is to achieve (semi-) continuous phase variation along the surface, which becomes important for largeangle operation, for which the required impedance profile has faster spatial variations. ${ }^{26}$ We have shown here that coarse sampling of the optimal profile provides reasonably good performance over wide angular and frequency ranges at visible frequencies. A possible way to overcome this limitation and gain higher efficiency (for larger retroreflection angle or more complicated functionalities) is to use a Pancharatnam-Berry phase, ${ }^{32}$ where the local variation of the surface phase is achieved through rotation of a subwavelength nanoantenna. Continuously varying surface elements can also provide a solution to eliminate the requirements on surface discretization. ${ }^{33}$

We believe that this approach is an effective replacement of conventional bulky optical elements, particularly for subwavelength gratings used in pulse shaping and spectrum splitting applications. The design is based on the Huygens-Fresnel principle, is scalable over different frequency ranges, and may be modified for achromatic retroreflection at multiple frequencies (or AOI). ${ }^{34,35}$ Similar designs may be explored at 
radio-frequencies to improve passive and active RFID tags. The control of the angle of reflection may also enable applications for light management in solar cells. The thin metasurface profile opens the possibility of spin coating or evaporating a thin absorber material on top of the metasurface and broadbandenhanced light trapping and absorption. ${ }^{14}$

\section{MATERIALS AND METHODS}

Derivation of the Reflection Coefficient. Following the Huygens-Fresnel principle to achieve the arbitrary scattering field distribution, the electromagnetic boundary conditions of the system in Figure 1C for s-polarized illumination read

$$
\begin{aligned}
& 2 \hat{z} \times\left.\left(\mathbf{H}_{\mathrm{i}}+\mathbf{H}_{\mathrm{s}}\right)\right|_{\sigma}=\left.Y_{\mathrm{e}}(x) \hat{y} \cdot\left(\mathbf{E}_{\mathrm{i}}+\mathbf{E}_{\mathrm{s}}\right)\right|_{\sigma} \\
& -2 \hat{z} \times\left.\left(\mathbf{E}_{\mathrm{i}}+\mathbf{E}_{\mathrm{s}}\right)\right|_{\sigma}=\left.Z_{\mathrm{m}}(x) y^{\wedge} \cdot\left(\mathbf{H}_{\mathrm{i}}+\mathbf{H}_{\mathrm{s}}\right)\right|_{\sigma}
\end{aligned}
$$

for any position $x$ on the inhomogeneous metasurface $\sigma$, where $Y_{\mathrm{e}}$ and $Z_{\mathrm{m}}$ are the effective surface electric admittance and surface magnetic impedance of the structure. ${ }^{12,14}$ Subscripts i and $\mathrm{s}$ indicate the incident and reflected waves, respectively. The local reflection coefficient of the metasurface $r(x)=$ $A(x) \mathrm{e}^{\mathrm{j} \varphi(x)}$ can be related to the effective properties of the surface for normal illumination as ${ }^{12,36}$

$$
r(x)=-\frac{2\left(\eta_{0}{ }^{2} Y_{\mathrm{e}}(x)-Z_{\mathrm{m}}(x)\right)}{\left(2+\eta_{0} Y_{\mathrm{e}}(x)\right)\left(2 \eta_{0}+Z_{\mathrm{m}}(x)\right)}
$$

Considering plane wave excitation at $\theta_{0}$ in the $x-z$ plane and ideal operation, i.e., unity retroreflection, the incident and scattered waves read

$$
\mathbf{E}_{\mathrm{i}, \mathrm{s}}=\hat{y} E_{0} \exp \left(\mathrm{i}\left(\hat{x} \cdot \mathbf{k}_{\mathrm{i}, \mathrm{s}} x+\hat{z} \cdot \mathbf{k}_{\mathrm{i}, \mathrm{s}} z\right)\right) ; \quad \eta_{0} \mathbf{H}_{\mathrm{i}, \mathrm{s}}=\hat{k}_{\mathrm{i}, \mathrm{s}} \times \mathbf{E}_{\mathrm{i}, \mathrm{s}}
$$

where $\mathbf{k}_{\mathrm{i}}=k_{0}\left(-\sin \left(\theta_{0}\right) \hat{x}+\cos \left(\theta_{0}\right) \hat{z}\right), \mathbf{k}_{\mathrm{s}}=-\mathbf{k}_{\mathrm{i}}$, and $k_{0}$ is the free-space wavenumber. Combining eqs 2,3 , and 4 the local reflection coefficient is found as eq 1 . Note that the local reflection coefficient is merely a nonlinear phase distribution imprinted on the reflected wave as $A(x)=1$. The phase profile for an ideal single-angle retroreflector with $\theta_{0}=35.7$ degrees is shown in Figure 2A. The local phase profile increasingly deviates from linear distribution for larger values of $\theta_{0}{ }^{26}$

Numerical Simulations. Numerical simulations throughout the paper were carried out by the $2 \mathrm{D}$ finite-element software COMSOL Multiphysics in the frequency-domain radio frequency module. Periodic ports and Floquet periodicity boundary conditions were used to model one unit cell of each metasurface. Ports were placed far enough from the metasurface such that all evanescent scattering modes are sufficiently weak. The ideal back reflector characterized in Figure 2 was modeled as a sheet admittance derived in Supplementary Note 1. The substrate and free space were meshed with maximum element sizes of 2 and $26 \mathrm{~nm}$, respectively. Perfect electric conductors were used in the ideal setup.

To model the fabricated device, we used the SEM images in Figure $3 \mathrm{~A}$ and $\mathrm{B}$ and estimated the dimensions as described in the fabrication process below. All materials were modeled as dispersive and lossy, and we used realistic values for the permittivities of silver and $\mathrm{SiO}_{x}$ from experimentally retrieved data sets. ${ }^{37,38}$ For $\mathrm{TiO}_{x}$, we measured the refractive index for a sample of $\mathrm{TiO}_{x}$ on a $\mathrm{Si}$ wafer by spectroscopic ellipsometry. A maximum element size of $20 \mathrm{~nm}$ was used for high-index $\mathrm{TiO}_{x}$ rods, and the remaining parts were meshed with a maximum element size of $28 \mathrm{~nm}$. A $200 \mathrm{~nm}$ thick silver layer was used as the back reflector, which we truncated with a perfectly matched layer to model a semi-infinite ground plane. The scattering parameters of the port were used to calculate the percentage of the power coupled toward each channel.

Fabrication Process. A 1-mm-thick Si wafer was coated with $200 \mathrm{~nm}$ of $\mathrm{Ag}$ and $20-30 \mathrm{~nm}$ of $\mathrm{SiO}_{x}$ by thermal evaporation. This protected mirror was then spin-coated with ZEP520a, a high-resolution positive tone resist, and Espacer $300 \mathrm{z}$ to improve the conductivity of the sample. Then the asymmetric grating was written by e-beam lithography using a $20 \mathrm{keV}$ beam. The patterned area was $1.5 \times 1.5 \mathrm{~mm}^{2}$ square, composed of stitched $100 \times 100 \mu \mathrm{m}^{2}$ write fields. The sample was then rinsed for $30 \mathrm{~s}$ in water to remove the Espacer, developed in pentylacetate for $45 \mathrm{~s}$, rinsed $15 \mathrm{~s}$ in a mixture of methyl isobutyl ketone and isopropyl alcohol (MIBK/IPA, 9:1), dipped into IPA, and transferred to ethanol. To prevent collapse of the fragile resist patterns, the sample was dried at the critical point. The lines were filled with $100 \mathrm{~nm}$ of $\mathrm{TiO}_{x}$ by e-beam evaporation followed by lift-off, which was done by dissolving the resist for $10 \mathrm{~min}$ in an ultrasonic bath in anisole. $\mathrm{TiO}_{x}$ was also evaporated directly on a $\mathrm{Si}$ wafer to allow a determination of the $\mathrm{TiO}_{x}$ dielectric function using spectroscopy ellipsometry, fitting the data using a Gaussian-Cauchy model. Completed dimensions were measured using a focused ion beam (FIB, FEI Helios Nanolab 600) to cut cross sections, with dimensions measured by electron micrographs. The metasurface consisted of repeating unit cells with a periodicity of $605 \mathrm{~nm}$. The taller line had a height of $100 \mathrm{~nm}$, a bottom width of $180 \mathrm{~nm}$, and a top width of $110 \mathrm{~nm}$. The narrower line, separated from the tall line by a gap of $123 \mathrm{~nm}$, had a height of $50 \mathrm{~nm}$ with bottom and top widths of 70 and $20 \mathrm{~nm}$, respectively (detailed dimensions are shown in Figure 3B).

Details of Optical Measurement. The coupling efficiency of the fabricated sample to the two orders was measured experimentally using a rotating stage, sweeping the angle of the incident plane wave across $\theta_{\text {in }}=-80$ degree to $\theta_{\text {in }}=80$ degree. For illumination, a collimated broadband beam from a laserdriven light source (EQ-99X, Energetiq) was sent through a linear polarizer and then focused onto the sample with a weak lens. The focal point on the sample had a diameter of $0.75 \mathrm{~mm}$. For excitation angles below 60 degrees the focus was smaller than the structure with an area of $1.5 \times 1.5 \mathrm{~mm}$; above this angle the light beam illuminated both the structured region and part of the adjacent mirrored substrate. This resulted in measured intensities of specular and first-order reflection with a slight contribution from the mirror. The intensity of the scattered power for the allowed scattering modes $s_{0}^{-}$and $s_{1}^{-}$was measured respectively for each degree using an optical power meter (PM100USB powermeter with S121C photodiode power sensor (Thorlabs)). The reflected beam was focused with a lens on the power sensor. To measure only the frequency ranges of interest, an optical filter (either 700, 750, or $850 \mathrm{~nm}$ center wavelength, $40 \mathrm{~nm}$ bandwidth, Andover Corporation) was placed in front of the lens of the power sensor.

\section{ASSOCIATED CONTENT}

\section{S Supporting Information}

The Supporting Information is available free of charge on the ACS Publications website at DOI: 10.1021/acsphotonics.6b00965. 
Details on the implementation of the ideal metasurface with continuous phase profile; discussion on the effects of surface discretization on the back-reflection efficiency; measurement results for a number of additional wavelengths; discussion on effects of fabrication errors on the performance of the device; details on the polarization response of the surface (PDF)

\section{AUTHOR INFORMATION}

\section{Corresponding Author}

*E-mail (A. Alù): alu@mail.utexas.edu.

ORCID

Andrea Alù: 0000-0002-4297-5274

Notes

The authors declare no competing financial interest.

\section{ACKNOWLEDGMENTS}

The authors acknowledge the assistance of Andries Lof and Dimitry Lamers in sample preparation, Brahim Ait Said for assistance with the measurement software, and Dion Ursem for technical support. We gratefully acknowledge Clara Osorio and Femius Koenderink for their support and productive conversations. This work was supported by the Royal Netherlands Academy of Sciences (KNAW), the U.S. Air Force Office of Scientific Research, the National Science Foundation, the Welch Foundation with grant No. F-1802, the Simons Foundation, and the European Research Council. The Dutch part of this work is part of the research program of the Netherlands Organization for Scientific Research (NWO), the European Research Council, and the Global Climate and Energy Project.

\section{REFERENCES}

(1) Kildishev, A. V.; Boltasseva, A.; Shalaev, V. M. Planar photonics with metasurfaces. Science 2013, 339, 1232009.

(2) Lin, D.; Fan, P.; Hasman, E.; Brongersma, M. L. Dielectric gradient metasurface optical elements. Science 2014, 345, 298-302.

(3) Yu, N.; Capasso, F. Flat optics with designer metasurfaces. Nat. Mater. 2014, 13, 139-150.

(4) Khorasaninejad, M.; Chen, W. T.; Devlin, R. C.; Oh, J.; Zhu, A. Y.; Capasso, F. Metalenses at visible wavelengths: Diffraction-limited focusing and subwavelength resolution imaging. Science 2016, 352, 1190-1194.

(5) Yu, N.; Genevet, P.; Kats, M. A.; Aieta, F.; Tetienne, J.-P.; Capasso, F.; Gaburro, Z. Light propagation with phase discontinuities: generalized laws of reflection and refraction. Science 2011, 334, 333337.

(6) Sun, S.; et al. High-Efficiency Broadband Anomalous Reflection by Gradient Meta-Surfaces. Nano Lett. 2012, 12, 6223-6229.

(7) Ni, X.; Kildishev, A. V.; Shalaev, V. M., Metasurface holograms for visible light, Nat. Commun. 2013, 4, DOI 10.1038/ncomms3807.

(8) Zheng, G.; Mühlenbernd, H.; Kenney, M.; Li, G.; Zentgraf, T.; Zhang, S. Metasurface holograms reaching $80 \%$ efficiency. Nat. Nanotechnol. 2015, 10, 308-312.

(9) Estakhri, N. M.; Alù, A. Ultra-thin unidirectional carpet cloak and wavefront reconstruction with graded metasurfaces. IEEE Antenn. Wireless Propag. Lett. 2014, 13, 1775-1778.

(10) Ni, X.; Wong, Z. J.; Mrejen, M.; Wang, Y.; Zhang, X. An ultrathin invisibility skin cloak for visible light. Science 2015, 349, $1310-1314$.

(11) Orazbayev, B.; Mohammadi Estakhri, N.; Beruete, M.; Alù, A. Terahertz carpet cloak based on a ring resonator metasurface. Phys. Rev. B: Condens. Matter Mater. Phys. 2015, 91, 195444.
(12) Pfeiffer, C.; Grbic, A. Metamaterial Huygens' surfaces: tailoring wave fronts with reflectionless sheets. Phys. Rev. Lett. 2013, 110, 197401.

(13) Pors, A.; Bozhevolnyi, S. I. Plasmonic metasurfaces for efficient phase control in reflection. Opt. Express 2013, 21, 27438-27451.

(14) Mohammadi Estakhri, N.; Alù, A. Manipulating optical reflections using engineered nanoscale metasurfaces. Phys. Rev. B: Condens. Matter Mater. Phys. 2014, 89, 235419.

(15) Hadad, Y.; Sounas, D.; Alù, A. Space-time gradient metasurfaces. Phys. Rev. B: Condens. Matter Mater. Phys. 2015, 92, 100304R.

(16) Shi, Y.; Fan, S. Dynamic non-reciprocal meta-surfaces with arbitrary phase reconfigurability based on photonic transition in metaatoms. Appl. Phys. Lett. 2016, 108, 021110.

(17) Wei, Z.; Li, H.; Wu, C.; Cao, Y.; Ren, J.; Hang, Z.; Chen, H.; Zhang, D.; Chan, C. T. Anomalous reflection from hybrid metamaterial slab. Opt. Express 2010, 18, 12119-12126.

(18) Su, X.; Wei, Z.; Wu, C.; Long, Y.; Li, H. Negative reflection from metal/graphene plasmonic gratings. Opt. Lett. 2016, 41, 348351.

(19) Deng, Z. L.; Zhang, S.; Wang, G. P. A facile grating approach towards broadband, wide-angle and high-efficiency holographic metasurfaces. Nanoscale 2016, 8, 1588-1594.

(20) Du, J.; Lin, Z.; Chui, S.; Lu, W.; Li, H.; Wu, A.; Sheng, Z.; Zi, J.; Wang, X.; Zou, S.; Gan, F. Optical beam steering based on the symmetry of resonant modes of nanoparticles. Phys. Rev. Lett. 2011, 106, 203903.

(21) Wu, C.; Arju, N.; Kelp, G.; Fan, J. A.; Dominguez, J.; Gonzales, E.; Tutuc, E.; Brener, I.; Shvets, G. Spectrally selective chiral silicon metasurfaces based on infrared Fano resonances, Nat. Commun. 2014, 5, DOI $10.1038 /$ ncomms 4892 .

(22) Rumpel, M.; Moeller, M.; Moormann, C.; Graf, T.; Ahmed, M. A. Broadband pulse compression gratings with measured $99.7 \%$ diffraction efficiency. Opt. Lett. 2014, 39, 323-326.

(23) Destouches, N.; Tishchenko, A.; Pommier, J.; Reynaud, S.; Parriaux, O.; Tonchev, S.; Abdou Ahmed, M. 99\% efficiency measured in the-1st order of a resonant grating. Opt. Express 2005, 13, 32303235.

(24) Flury, M.; Tishchenko, A. V.; Parriaux, O. The leaky mode resonance condition ensures $100 \%$ diffraction efficiency of mirrorbased resonant gratings. J. Lightwave Technol. 2007, 25, 1870-1878.

(25) Astilean, S.; Lalanne, P.; Chavel, P.; Cambril, E.; Launois, H. High-efficiency subwavelength diffractive element patterned in a highrefractive-index material for $633 \mathrm{~nm}$. Opt. Lett. 1998, 23, 552-554.

(26) Mohammadi Estakhri, N.; Alù, A. Wavefront Transformation with Gradient Metasurfaces. Phys. Rev. X 2016, 6, 041008.

(27) Mohammadi Estakhri, N.; Alù, A. Recent progress in gradient metasurfaces. J. Opt. Soc. Am. B 2016, 33, A21-A30.

(28) Rumsey, V. Reaction concept in electromagnetic theory. Phys. Rev. 1954, 94, 1483.

(29) Whitman, G.; Schwering, F. Reciprocity identity for periodic surface scattering. IRE Trans. Antennas Propag. 1979, 27, 252-254.

(30) Lloyd, J. A Brief History of Retroreflective Sign Face Sheet Materials; The Retroreflective Equipment Manufacturers Association: Lancashire, UK, http://www.rema.org.uk/. Accessed February 15, 2010.

(31) Yang, Y.; Moitra, P.; Kravchenko, I. I.; Briggs, D. P.; Valentine, J.; Wang, W. Dielectric Meta-Reflectarray for Broadband Linear Polarization Conversion and Optical Vortex Generation. Nano Lett. 2014, 14, 1394-1399.

(32) Hasman, E.; Kleiner, V.; Biener, G.; Niv, A. Polarization dependent focusing lens by use of quantized Pancharatnam-Berry phase diffractive optics. Appl. Phys. Lett. 2003, 82, 328-330.

(33) Li, Z.; Palacios, E.; Butun, S.; Aydin, K. Visible-Frequency Metasurfaces for Broadband Anomalous Reflection and HighEfficiency Spectrum Splitting. Nano Lett. 2015, 15, 1615-1621.

(34) Aieta, F.; et al. Multiwavelength achromatic metasurfaces by dispersive phase compensation. Science 2015, 347, 1342-1345. 
(35) Deng, Z.; Zhang, S.; Wang, G. P. Wide-angled off-axis achromatic metasurfaces for visible light. Opt. Express 2016, 24, 23118-23128.

(36) Holloway, C. L.; Mohamed, M. A.; Kuester, E. F.; Dienstfrey, A. Reflection and transmission properties of a metafilm: With an application to a controllable surface composed of resonant particles. IEEE Trans. Electromagn. Compat. 2005, 47, 853-865.

(37) Malitson, I. Interspecimen Comparison of the Refractive Index of Fused Silica. J. Opt. Soc. Am. 1965, 55, 1205-1209.

(38) Rakić, A. D.; Djurišić, A. B.; Elazar, J. M.; Majewski, M. L. Optical properties of metallic films for vertical-cavity optoelectronic devices. Appl. Opt. 1998, 37, 5271-5283. 